\title{
The Anomalous Effect of Quantum Interference in Organic Spin Filters
}

Ashima Bajaj, ${ }^{\dagger}$ Prabhleen Kaur,$^{\dagger}$ Aakanksha Sud,$^{\dagger}$ Marco Berritta, ${ }^{\dagger}$ and Md. Ehesan Ali*,

$\dagger$ Institute of Nano Science and Technology, Phase 10, Sector-64, Mohali Punjab-160062, India

$\ddagger$ Department of Physics and Astronomy, Uppsala University, Box 516, 75120 Uppsala, Sweden

E-mail: ehesan.ali@inst.ac.in 


\begin{abstract}
The molecular topology in the single-molecular nano-junctions through which the de-Broglie wave propagates plays a crucial role in controlling the molecular conductance. The enhancement and reduction of the conductance in para and meta connected molecules due to constructive and destructive Quantum Interference (QI) respectively are quite well established. Herein, we investigated the effect of localized spin centers on spin transportation using organic radicals as molecular junctions. The role of the localized spins on the QI as well as on spin filtering capability is investigated employing density functional theory in combination with non-equilibrium Green's function (NEGFDFT) techniques. Various organic radicals including nitroxy (NO), phenoxy (PO*) and methyl $\left(\mathrm{CH}_{2}\right)$ attached to the central benzene ring of pentacene with different terminal connections (para and meta) to gold electrodes are examined. Due to more obvious QI effects, para connected pentacene is found to be more conductive than meta one. Surprisingly, on incorporating a radical center, along with spin filtering, a significant reversal of QI effects is observed which manifests itself in such a way that the conductance of meta coupled radicals is found to be more than para by two orders of magnitude. The anomaly in QI patterns induced by radical center is analyzed and discussed in terms of orbital and structural perspectives.
\end{abstract}

\title{
1 Introduction
}

On a road towards miniaturization of electronic components, the community has expanded its roots to molecular electronics. ${ }^{1,2}$ Over the past decades, molecular electronics has allied with organic spintronics for various technological applications including high density memory storage, magnetic logic devices, magnetic sensors and so on. ${ }^{3,4}$ Various aspects of molecular spintronics which involve manipulation of electron spin, spin filtering, spin tunneling, spin transport across the interface have been an extensive area of research from the past many years. ${ }^{5,6}$ Among them, spin filters are the devices that enable to produce spin-polarized 
current by preferentially favoring the transport of electron of one spin orientation over the other. Organic molecules are the most appealing candidates for such components owing to the weak spin-orbit and hyperfine interactions. The use of organic radicals as components of spin filters was first reported by Herrmann and coworkers. ${ }^{7,8}$ The degeneracy splitting of spin-up and spin-down orbitals in organic radicals bring the MO level of one spin orientation closer to the Fermi level of electrodes and thus resulting in higher conductance of one spin type over the other. ${ }^{9}$ Followed by this, Smeu et al. investigated the spin filtering efficiency of various $\sigma$ and $\pi$ type radical systems. ${ }^{10}$ Shil et al. came up with the use of high spin organic diradical as a component of spin filter. ${ }^{11}$

The capability to tailor the electron spin with an enhanced control over electron transport in organic molecular materials has become a biggest challenge for molecular spintronics. ${ }^{12}$ Of the various quantum effects that can be exploited in phase coherent regime, Quantum Interference (QI) plays a fundamental role in controlling the current through single molecule devices. ${ }^{13}$ QI effects are associated with coherent superposition of de Broglie waves traversing along multiple paths ${ }^{14}$ or only frontier molecular orbitals through a molecule. ${ }^{15,16}$ The most striking feature of QI is the appearance of sharp dips in transmission close to Fermi energy which appear when the phases of the electron traversing along different pathways of the molecule exactly cancel each other, thus leading to destructive quantum interference (DQI) and an obvious decrease in conductance. On the other hand, interference manifests itself in a constructive (CQI) way when the electron waves traversing along different pathways are in-phase with each other. The presence/absence of sharp dips featuring DQI has been demonstrated extensively in recent years in various systems such as cross-conjugated molecules ${ }^{17,18}, \pi$-conjugated molecules with different connectivity ${ }^{19}$, effect of introduction of heteroatoms. ${ }^{20,21}$ Recently, Tao and coworkers have experimentally probed the charge transport by tuning the QI in para and meta connected molecules with an electrochemical gate voltage. $^{22}$

A single benzene molecule where the electrodes are attached meta with respect to each 
other is expected to exhibit low conductance due to destructive quantum interference, while para-coupled analogue is predicted to be a better conductor. ${ }^{23}$ This switching from CQI to DQI on changing the connectivity has been established starting from a single benzene molecule to various polycyclic hydrocarbons. ${ }^{24,25}$ But only few reports have described QI effects in systems exhibiting spin polarization. ${ }^{26-29}$ Recently, Li et al. proposed a mechanism for tuning the spin filtering ratio based on QI effects in a nickel based junction. ${ }^{30}$ Ratner and coworkers have demonstrated that how DQI features can be controlled by addition of a donor or acceptor group to the polyacetylene chain bridging graphene nanoribbons (ZGNR). ${ }^{31} \mathrm{But}$ the role of QI in tuning the overall conductance and the spin filtering efficiency of organic radical molecular junction, which is expected to be the most efficient spin filter has not been explored yet.

(a)

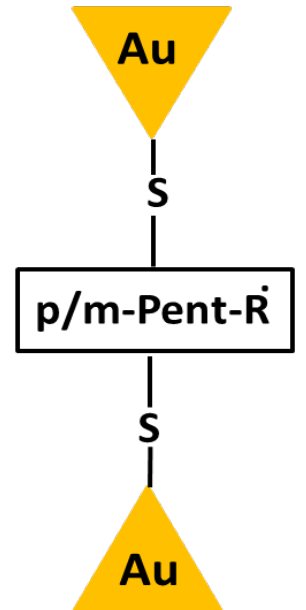

(b)
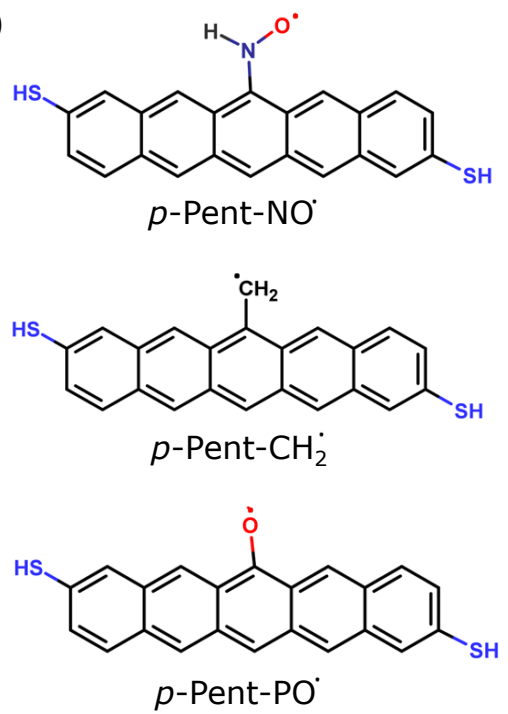
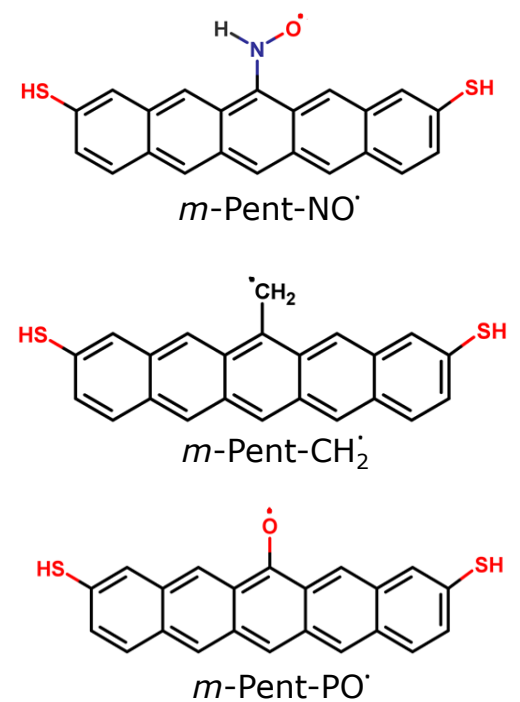

Figure 1: (a)Pentacene based radicals with para $(p)$ and meta $(m)$ coupling of terminal benzene rings of pentacene to gold electrodes via thiol (S) anchoring group. (b) Molecular structures of investigated pentacene based radicals where Nitroxy (NO), Methyl $\left(\mathrm{CH}_{2}{ }^{\circ}\right)$ and Phenoxy (PO) radicals are attached to central benzene ring of pentacene core.

In this study we probed the spin polarized transport using organic radicals as spin selective transmission source. Our aim is to investigate the dependence of conductance on the topological structure of the radicals, i.e., how combinations of para and meta couplings affect the spin polarized transport through the organic spin filters. Molecular bridges based on 
pentacene as central bridging unit are considered. We examined how the transition from CQI to DQI observed in parent pentacene on moving from para to meta connection is affected by attaching a radical across the pentacene core. Organic radicals including Nitroxy (NO), Phenoxy (PO) and Methyl $\left(\mathrm{CH}_{2}{ }^{\circ}\right)$ are attached to central benzene ring of pentacene core, which is coupled to gold electrodes via thiol linkers as shown in Fig. 1. The electron transport in molecular junctions has been explored using a fully self-consistent non-equilibrium Green's

function (NEGF) approach with periodic models. ${ }^{32}$ However, in this DFT based approach, the electron transport through molecular junction is assumed to be coherent and elastic, where inelastic corrections due to electron-phonon interactions are not taken into account. Apart from this, the unavoidable self-interaction errors (SIEs) associated with exchange-correlation functionals of DFT results in underestimated energy gaps between empty and occupied orbitals which may cause large currents and overestimated conductance as compared to experimental values. ${ }^{33,34}$ This can be improved by using more accurate hybrid functionals which not only improve self-interaction correction but also provide better orbital energies. Thus, in an alternative approach, we employed density functional beyond the commonly employed GGA approximation using a non-self-consistent NEGF approach but paying the price of describing the electrodes as a small finite cluster of metal atoms.

\section{Computational Details}

The spin dependent charge transport characteristics of all the molecules are investigated using NEGF-DFT technique as implemented in SIESTA package. ${ }^{35}$ The system studied consists of pentacene based radicals presented in Fig. 1b bridged between two gold electrodes in a two probe transport structure, consisting of three parts: left electrode, central scattering region and right electrode, as shown in Fig. 2. In the first step, isolated molecules (shown in Fig. 1b) are optimized including the two S-H bonds at both the ends using B3LYP/def2-TZVP method in $O R C A{ }^{36}$ The optimized pentacene-radical systems are then placed between two gold 
electrodes by removing the terminal H-atoms and forming S-Au bonds. The Au electrodes are modeled as $\mathrm{Au}(111) 5 \times 5$ surface with 3 layers in transport direction. The scattering region is composed of molecule connected to the conical gold junction containing four $\mathrm{Au}$ atoms on both sides and an additional $5 \times 5$ layer to replicate the bulk electrode. Geometry optimization is then performed for Au-Pentacene-Au junction with DFT as implemented in SIESTA code, with PBE exchange correlation functional. ${ }^{35}$ During the optimization, all the gold atoms were fixed in their bulk lattice positions and only molecule is allowed to relax within the junction. In the relaxed junction, Au-S distance is found to vary from 2.35 to $2.40 \AA$. Further, an additional charge transfer was induced by S-atom attached to Au atoms which made the gold lead magnetic by behaving as radical center with an unpaired electron. To circumvent this additional charge transfer from S-atom to electrode due to the formation of S(radical), a net charge of -2 is given to the whole Au-molecule-Au junction. The electronic charge transport properties are obtained using TranSIESTA code, which allows a periodic description of electrodes. ${ }^{37}$ Norm-conserving pseudopotentials are used to describe the core electrons, double- $\zeta$ polarized (DZP) basis set is used to describe the valence electrons of molecular atoms and gold atoms. ${ }^{38}$ The generalized gradient approximation (GGA) is used for exchange correlation function. A mesh cutoff of 300 Ry is used with Brillouin zone sampling using $3 \times 3 \times 10$ k-points. All the calculations are converged by using the conjugate gradient (CG) algorithm. The convergence tolerance in the density matrix is set to $10^{-4} \mathrm{eV}$, whereas the residual forces on all the atoms are lesser than $0.02 \mathrm{eV} / \AA$.

Since, GGA functional show limitations in calculations of orbital energies. Thus, to go beyond GGA approximation, we also performed non-periodic calculations using hybrid density functional, following an alternative cluster approach. In this approach, the transmission functions are obtained using a NEGF based post processing tool ARTAIOS. ${ }^{39,40}$ The optimized pentacene-radical systems are first placed between two $\mathrm{Au}_{9}$ clusters, approximating the electrode surface, fixing Au-S distance to $2.40 \AA$. In the next step, single point calculations are carried out for cluster-molecule-cluster system at B3LYP/LANL2DZ level using Gaussian 


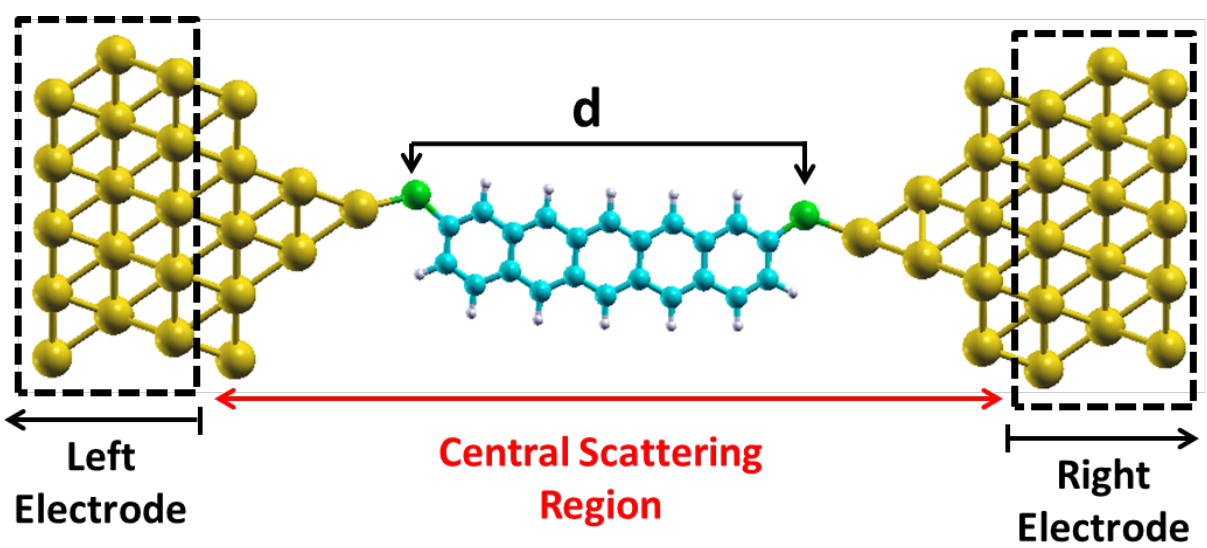

Figure 2: Device setup of Au-Molecule-Au junction with pentacene bridge coupled to $\mathrm{Au}(111)$ electrodes via sulphur atoms. The central scattering region consists of conical gold junction with an additional $5 \times 5$ layer of $\mathrm{Au}$ and central molecule. Semi-infinite left and right electrodes extend up to $\mathrm{z}= \pm \infty$.

09 to extract the hamiltonian and overlap matrices needed for transport calculations. A rather simpler wide-band limit approximation is employed for electrode, which assumes that density of states (DOS) of electrode is independent of the energy. A constant value of 0.036 $e V^{-1}$, taken from the literature, is used for the DOS of electrode. ${ }^{8}$

The implementation of NEGF formalism in combination with DFT (NEGF-DFT) has been thoroughly described in literature. ${ }^{32,41}$ To briefly summarize, the retarded Green's function at energy $\mathrm{E}$ is obtained by inverting the Hamiltonian matrix as

$$
G(E)=\left((E+\iota \eta) S-H-\Sigma_{L}-\Sigma_{R}\right)^{-1}
$$

where $\mathrm{H}$ and $\mathrm{S}$ are the Hamiltonian and the Overlap matrices for molecular region as determined by DFT. $\iota \eta$ is the infinitesimal imaginary part added in energy. $\Sigma_{L / R}$ are the self-energies that account for the effect of left and right electrode on central scattering region. Self energy is a complex quantity whose real part represents shift of energy levels and the imaginary part accounts for the level broadening matrix:

$$
\Gamma_{L / R}=\iota\left(\Sigma_{L / R}-\Sigma_{L / R}^{\dagger}\right)
$$


The quantity of interest here is electron transmission function, $T_{\sigma}(E)$, which represents the probability with which an electron of spin $\sigma$ (up/down) and energy E can transmit from the left electrode to right electrode, through central scattering region. According to the Landauer-Büttiker formalism, the transmission function can be calculated as the trace of the product of coupling matrices $\Gamma_{L / R}$ and the Green's function of the central scattering region

$$
T_{\sigma}(E)=\operatorname{Tr}\left(\Gamma_{R} G \Gamma_{L} G^{\dagger}\right)_{\sigma}
$$

The zero bias conductance is then obtained as

$$
G=G_{\circ} T\left(E_{F}\right)
$$

where $G_{\circ}=2 e^{2} / \mathrm{h}$ is the quantum of conductance.

The spin filtering efficiency (SFE) ${ }^{31}$ at the Fermi level is then given by

$$
S F E=\frac{\left|T_{u p}\left(E_{F}\right)-T_{\text {down }}\left(E_{F}\right)\right|}{T_{u p}\left(E_{F}\right)+T_{\text {down }}\left(E_{F}\right)}
$$

The transmission spectra extracted from periodic models is interpreted in terms of Transmission Eigenchannels (EC) and Molecular Projected Self-consistent Hamiltonian (MPSH states) which is obtained by projecting the Hamiltonian on the molecular orbitals of the central molecule. The wave functions of EC and MPSH states are obtained by using Inelastica package. ${ }^{42}$ For the transmission spectra associated to cluster approach, local transmission maps are obtained using ARTAIOS code which describe the conduction pathway of the electrons at certain energies. ${ }^{43}$ 


\section{Results and Discussions}

\subsection{QI in Parent Non-magnetic Acenes}

The low transmission caused due to QI effects in para and meta connected benzene is well understood. ${ }^{23,44}$ Due to equal path lengths, interference manifests itself in a constructive way leading to high transmission probability and high conductance in $p$-Benzene. However, in $m$-Benzene, the phase difference between two pathways is equal to $\pi$, which gives rise to destructive QI and hence less conductance in $m$-Benzene. ${ }^{45-47}$ To understand the modification in the DQI pattern brought about by increasing the molecular length, we calculated the transmission spectra for a series of acenes $(\mathrm{n}=1$ to 5$)$, considering both para and meta linkage of the terminal benzene rings. Fig. 3 shows the evolution of transmission spectra, extracted using periodic models. For all the para and meta connected acenes ( $n=1$ to 5 ), the alignment of $\mathrm{HOMO}$ with respect to the Fermi level of electrode is virtually the same (within $0.2 \mathrm{eV}$ ) for both para and meta connected acenes in junctions (shown in Fig. 3 by arrows marked below Fermi level). However, the electronic structure of isolated acene molecules reveal that HOMO of the molecule is shifted by 0.69 and $0.92 \mathrm{eV}$ for para and meta connection respectively on moving from benzene to pentacene (See SI Table S1 and Fig. S2). Thus, pointing towards the occurrence of HOMO level pinning in acene based molecular junctions. The evidence of such a pinning has also been provided in recent studies. ${ }^{48,49}$ Further, the evolution of resonance peaks arising from LUMO (shown in Fig. 3 by arrows marked above Fermi level) reveals that LUMO tends to get closer to the Fermi level on moving from benzene to pentacene, thus promoting the reduction of HOMO-LUMO gap on increasing length of acene molecules. Apart from this, for benzene case, the HOMO and LUMO resonances are also accompanied by several other peaks appearing between HOMO-LUMO gap. The projected density of state (PDOS) of the molecular atoms and gold lead (shown in SI) reveals that these additional peaks do not resemble to any of the electronic state of the benzene 
molecule but are induced due to the spurious lead-lead and cross-lead interactions. However, with the increasing molecular length, the appearance of lead states subsequently decreases and eventually disappears upon approaching tetracene due to the larger spatial separation distance between the leads.

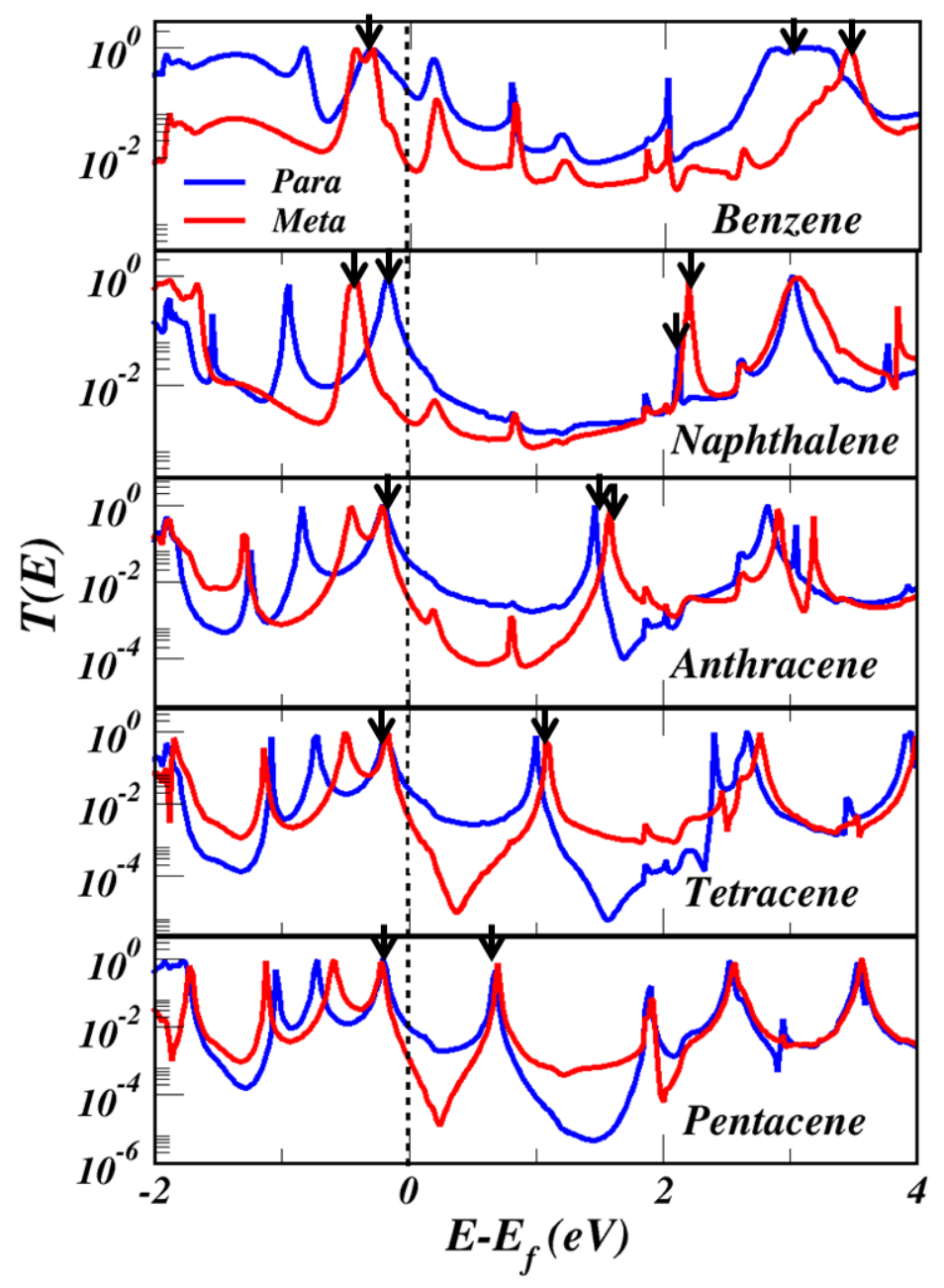

Figure 3: Transmission spectra obtained from periodic models for para and meta connected acenes, $n=1-5$, where blue and red curves correspond to acenes with para and meta connections respectively. The vertical dotted line denotes the position of Fermi level. The arrows marked over the peaks below and above Fermi energy corresponds to HOMO and LUMO resonance respectively.

Further, the transmission spectra of para and meta connected acenes reveals that the position of resonance peaks which correspond to the conduction channel through molecular orbitals is identical for both para and meta connections. This is also illustrated by energy level 
diagram (Fig. S2 and Fig. S4) wherein both para and meta connected acenes exhibit almost similar energies of HOMO and LUMO in molecular junction as well as in isolated gas phase. Along with the resonance peaks, the position of the dips residing between the resonance peaks arising due to the absence of the molecular states is also identical irrespective of the para and meta connection. Typically, the molecular junctions containing meta connected molecule results in destructive QI which is distinguished by a sharp dip between HOMO and LUMO resonances. However, interference manifests itself in a constructive way in para connected molecule wherein instead of a sharp peak, CQI results in a usual dip featuring absence of molecular states. ${ }^{50}$ Fig. 3 also reveals that for all the $n=1$ to 5 acenes, the dip residing between HOMO and LUMO resonances near Fermi energy is always sharper for meta connected acenes than para ones. This clearly indicates that the electron transmission through meta connected acenes is governed by DQI near Fermi energy leading to low conductance. On the other hand, electron transmission occurs constructively through para connections. Moreover, the sharpness of this dip for meta connected acenes further manifests itself with increasing molecular length in such a way that the difference between the dips of para and meta connected pentacene shoots to the maxima of two orders of magnitude. The evolution of DQI trends on moving from benzene to pentacene is also evident in the transmission spectra obtained using cluster approach, shown in SI, demonstrating the intense signatures of QI in case of pentacene. Thus owing to the most evident signatures of QI in pentacene, we consider pentacene as a central bridging unit for the spin-polarized radical molecular junctions discussed in subsequent subsections.

\subsection{QI in Pentacene based Radicals}

The spin resolved transmission spectra, extracted from periodic models, for parent pentacene and pentacene based radical systems under study is shown in Fig. 4. For parent pentacene, the dip residing between HOMO and LUMO resonances near Fermi energy drops to $\sim 10^{-5}$ in $m$-Pent, while owing to constructive interference, $p$-Pent yields a dip of the order of $10^{-3}$. 
Further, being a closed shell molecule bearing no magnetic center, parent pentacene exhibits no spin polarization. However, for radical systems, the spin-up and spin-down transmission spectra differs significantly due to splitting of the degeneracy caused by an unpaired electron.

The transmission spectra of all the radical species under study (shown in Fig. 4) reveals that for all the meta connected radicals, the maximum achieved value of transmission peaks is about 0.95 for both the spin orientations. However, when the lead positions change from meta to para, the amplitude of transmission peaks decreases to around 0.10 for both spin configurations. For spin-up orientation, the sharp dip ascribed to DQI in $m$-Pent is shifted to higher energy of about $0.80 \mathrm{eV}$ in meta connected radicals. As a result, the suppression effect imposed by DQI dip is much weaker yielding higher conductance of the order of $10^{-2}$ near Fermi energy. In contrast, for para-connected radicals, a significant decrease in the transmission amplitude of HOMO and LUMO resonance peaks results in an inefficient transmission and decreased conductance of the order of $10^{-4}$. This reversal of para-meta conductance upon attachment of radical is found to be even more prominent for spin-down orientation. For para connected radicals, DQI features with sharp dips of the order of $10^{-6}$ are evidently observed near Fermi energy except $p$-Pent-PO: This clearly indicates the modulation from CQI to DQI in para connection upon attachment of localized radical center. On contrary, for meta connected radicals, the minima of the dip residing between spin-down HOMO and LUMO resonances at $\sim-0.20 \mathrm{eV}$ is observed to be the order of $10^{-3}$, with no absolute DQI features as observed in parent $m$-Pent. This again points towards the crossover from DQI to CQI in meta connection by attaching a localized radical center. In nutshell, one of the spin orientation yields same QI pattern as of non-magnetic pentacene but accompanied by the change in order of para-meta conductance, while the other spin orientation results in complete reversal of QI effects as observed in parent pentacene.

The zero bias conductance illustrated in Table 1 also reveals that switching from para to meta connectivity causes the total conductance $\left(\mathrm{G}_{\text {total }}\right)$ to drop by an order of magnitude in parent pentacene core. In contrast, for the pentacene based radicals, the same operation 


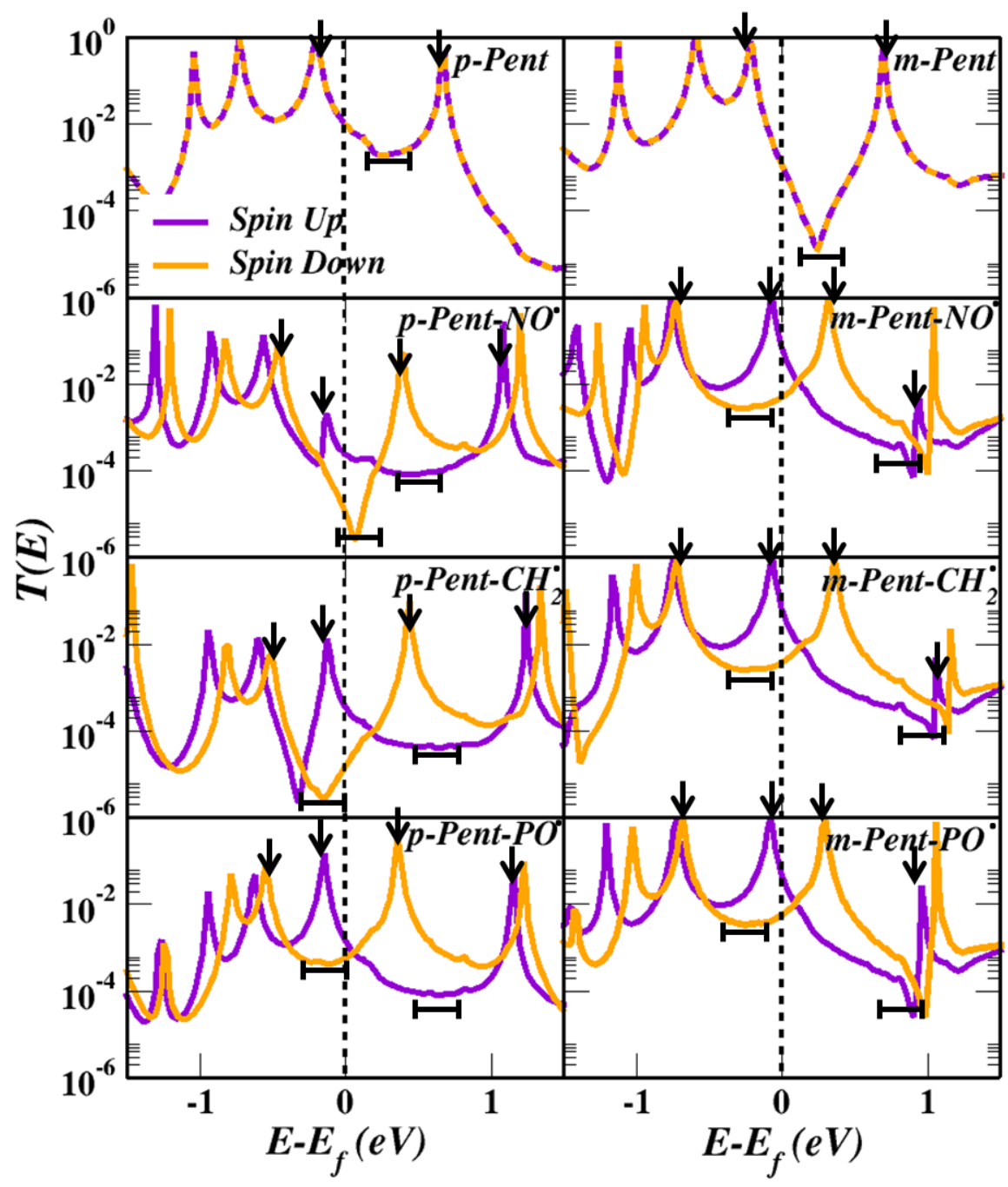

Figure 4: Spin resolved transmission spectra obtained from periodic models, for para and meta connected pentacene (top panel) and pentacene based radicals ( $\mathrm{NO}^{\circ} \mathrm{CH}_{2}^{\circ}$, PO) where spin-up and spin-down transmission is denoted by purple and orange respectively. The vertical dotted lines denote the position of Fermi level. The arrows marked over the peaks below and above Fermi level denote HOMO and LUMO resonance respectively wherein the arrows pointing towards purple peaks denote spin-up resonance and those towards orange peaks are for spin-down resonance. The dips residing between HOMO and LUMO resonances are also marked for both the spin channels. 
causes the conductance of both spin channels as well as total conductance to increase by two orders of magnitude for $\mathrm{NO}^{\circ}$ and $\mathrm{CH}_{2}$ and one order of magnitude for $\mathrm{PO}$. Thus, indicating the strong reversal of QI effects observed in non-magnetic pentacene by attached radical moieties, thereby making meta connected radicals more conductive than para ones. The reversal of para/meta order of conductance can also be seen clearly from sharp decrease in para/meta conductance ratio, which is of the order of $10^{0}$ for parent pentacene, but has decreased to $10^{-3}$ for $\mathrm{NO}^{\bullet}$ and $\mathrm{CH}_{2}$ radicals and $10^{-2}$ for $\mathrm{PO}^{*}$ radical. Herrmann et al. also anticipated the change in para/meta conductance ratio on introducing a radical group or any closed shell substituent that participates in $\pi$ electron system, however, no any clear correlation was made based on QI effects. ${ }^{8}$

Table 1: Calculated conductance for spin-up $\left(\mathrm{G}_{\mathrm{up}}\right)$ and spin-down $\left(\mathrm{G}_{\text {down }}\right)$ channels, total conductance $\left(G_{\text {total }}\right)$ in units of $G_{0}$ and spin filtering efficiency (SFE) for para and meta connected pentacene based radicals.

\begin{tabular}{|c|c|c|c|c|c|c|}
\hline \multirow{2}{*}{ Molecule } & \multirow{2}{*}{ Anchoring } & \multicolumn{3}{|c|}{$\mathrm{G}\left(G_{\circ}\right)$} & \multirow{2}{*}{$\operatorname{SFE}(\%)$} & \multirow{2}{*}{ para/meta $\left(G_{\text {total }}\right)$} \\
\hline & & $\mathrm{G}_{\text {up }}$ & $\mathrm{G}_{\text {down }}$ & $\mathrm{G}_{\text {total }}$ & & \\
\hline \multirow{2}{*}{ Pent } & para & $9.65 \times 10^{-3}$ & $9.66 \times 10^{-3}$ & $1.93 \times 10^{-2}$ & 0.0 & $8.53 \times 10^{0}$ \\
\hline & meta & $1.13 \times 10^{-3}$ & $1.13 \times 10^{-3}$ & $2.26 \times 10^{-3}$ & 0.0 & \\
\hline \multirow{2}{*}{ Pent-NO } & para & $2.39 \times 10^{-4}$ & $1.10 \times 10^{-5}$ & $2.50 \times 10^{-4}$ & 91.2 & $3.37 \times 10^{-3}$ \\
\hline & meta & $7.01 \times 10^{-2}$ & $4.82 \times 10^{-3}$ & $7.49 \times 10^{-2}$ & 87.0 & \\
\hline \multirow{2}{*}{ Pent- $\mathrm{CH}_{2}{ }^{\circ}$} & para & $3.54 \times 10^{-4}$ & $1.48 \times 10^{-5}$ & $3.68 \times 10^{-4}$ & 92.1 & $5.13 \times 10^{-3}$ \\
\hline & meta & $6.80 \times 10^{-2}$ & $3.70 \times 10^{-3}$ & $7.17 \times 10^{-2}$ & 82.6 & \\
\hline \multirow{2}{*}{ Pent-PO } & para & $1.42 \times 10^{-3}$ & $5.54 \times 10^{-4}$ & $1.97 \times 10^{-3}$ & 43.8 & $3.68 \times 10^{-2}$ \\
\hline & meta & $4.80 \times 10^{-2}$ & $5.48 \times 10^{-3}$ & $5.34 \times 10^{-2}$ & 79.5 & \\
\hline
\end{tabular}

Apart from the observed anomaly in QI effects in the radical molecular junctions, all the radical systems results in significant spin polarization for both para and meta connections. As illustrated by transmission spectra, shown in Fig. 4, a conduction peak is observed just below Fermi energy for spin-up orientation (marked by arrow over purple peak below Fermi energy). This peak pinned to Fermi energy for all the radical cases, corresponds to the non-bonding character of singly occupied molecular orbital (SOMO) of the radical species. While for spin-down orientation no such pinning is observed wherein HOMO and LUMO resonance peaks are positioned symmetrically below and above Fermi energy respectively. Further, the 
spin filtering efficiency (SFE) calculated using Eq. 5 is tabulated in Table 1. A perfect spin filtering of more than $75 \%$ is achieved for all para and meta connected radicals while for $p$-Pent-PO' a moderate SFE of $43.8 \%$ is obtained. The spin density plots (shown in SI) reveals that for all the radical systems under study, the spin density is not only localized on the radical centers, but is completely delocalized on entire pentacene backbone. The high SFE facilitated by the extensive delocalization of spin density on the bridging pentacene molecule is also observed in one of our recent work. ${ }^{51}$ Apart from this, for $\mathrm{NO}^{*}$ and $\mathrm{CH}_{2}$ radicals, a spin moment of 0.195 and $0.115 \mu_{B}$ was found to be localized on $\mathrm{NO}^{\circ}$ and $\mathrm{CH}_{2}{ }^{\circ}$ centers respectively, however, for PO case, only $0.051 \mu_{B}$ spin moment was found to be localized on the $\mathrm{O}^{*}$ radical center. The spurious over-delocalization of spin density in $\mathrm{PO}^{\circ}$ case could be one of the reason of its exceptional behavior with no DQI feature in spin-down transmission spectra of $p$-Pent-PO as observed in other two radical cases.

Further, the anomaly observed in QI effects by the radical centers is strongly supported by calculated zero-bias conductance of the hydroxy or methyl derivatives of the radical systems shown in Fig. 1b, deliberately making them non-radical species. The obtained zero bias conductance of para and meta connected non-radical counterparts tabulated in Table 2 is found to be concordant with pentacene i.e. para is more conductive than meta. Thus, supporting our observation that radical unpaired electron is playing an anomalous role in tuning the conductance of molecular junction with localized spin centers.

Finally yet importantly, the inversion of para/meta conductance relation on attaching a radical is also evident from the transmission spectra obtained from cluster approach employing hybrid B3LYP functional (shown in SI). Within this approach, much better electronic structures of the scattering regions are produced. Owing to the higher HOMOLUMO gap provided by the use of B3LYP as compared to the PBE functional, broader dips are observed near Fermi energy. Thus, clearly revealing higher conductance of meta connected radicals as compared to the para ones. 
Table 2: Calculated conductance for spin-up $\left(\mathrm{G}_{\mathrm{up}}\right)$ and spin-down $\left(\mathrm{G}_{\text {down }}\right)$ channels, total conductance $\left(G_{\text {total }}\right)$ in units of $G_{\circ}$ for para and meta connected pentacene based non-radicals.

\begin{tabular}{|c|c|c|c|c|c|}
\hline \multirow{2}{*}{ Molecule } & \multirow{2}{*}{ Anchoring Position } & \multicolumn{3}{|c|}{$\mathrm{G}\left(G_{\circ}\right)$} & \multirow{2}{*}{ para/meta $\left(G_{\text {total }}\right)$} \\
\hline & & $\mathrm{G}_{\text {up }}$ & $\mathrm{G}_{\text {down }}$ & $\mathrm{G}_{\text {total }}$ & \\
\hline \multirow{2}{*}{ Pent } & para & $9.65 \times 10^{-3}$ & $9.66 \times 10^{-3}$ & $1.93 \times 10^{-2}$ & \multirow[t]{2}{*}{$8.53 \times 10^{0}$} \\
\hline & meta & $1.13 \times 10^{-3}$ & $1.13 \times 10^{-3}$ & $2.26 \times 10^{-3}$ & \\
\hline \multirow{2}{*}{ Pent-NOH } & para & $8.54 \times 10^{-3}$ & $8.55 \times 10^{-3}$ & $1.70 \times 10^{-2}$ & \multirow[t]{2}{*}{$11.2 \times 10^{0}$} \\
\hline & meta & $7.32 \times 10^{-4}$ & $7.79 \times 10^{-4}$ & $1.51 \times 10^{-3}$ & \\
\hline \multirow{2}{*}{ Pent- $\mathrm{CH}_{3}$} & para & $9.53 \times 10^{-3}$ & $9.54 \times 10^{-3}$ & $1.90 \times 10^{-2}$ & \multirow[t]{2}{*}{$7.66 \times 10^{0}$} \\
\hline & meta & $1.20 \times 10^{-3}$ & $1.28 \times 10^{-3}$ & $2.48 \times 10^{-3}$ & \\
\hline \multirow{2}{*}{ Pent-POH } & para & $1.18 \times 10^{-2}$ & $1.18 \times 10^{-2}$ & $2.36 \times 10^{-2}$ & \multirow[t]{2}{*}{$3.02 \times 10^{0}$} \\
\hline & meta & $3.81 \times 10^{-3}$ & $4.00 \times 10^{-3}$ & $7.80 \times 10^{-3}$ & \\
\hline
\end{tabular}

\subsection{Molecular Orbitals Perspectives in Electron Transport}

Since QI effects are purely quantum mechanical effects and are associated to the molecular properties in junction such as its structure and symmetry. Thus, to elucidate the origin of the significant difference in para/meta conductance ratio in radical molecular junctions, we investigated the electronic properties of the molecule in junction. Since the coupling between the electrode and molecule will also modify the molecular orbitals (MOs) through rehybridization. Thus, the modified MOs are visualized by molecule projected self-consistent Hamiltonian (MPSH) eigenstates. ${ }^{52,53}$ The transmission characteristics are further investigated in terms of the transmission eigenchannels and local transmissions, as discussed in the subsequent subsections.

\subsubsection{Frontier MPSH Orbitals}

MPSH orbitals represent the modified molecular orbitals (MOs) and their energy levels in the presence of surrounding electrodes. ${ }^{53}$ To predict QI in molecular junctions, a variety of rules such as magic ratio rules $(\mathrm{MRR})^{54}$ and the one based on a graphical scheme in atomic orbital (AO) basis have been formulated in the literature. ${ }^{24}$ Another such scheme is based on MO perspective wherein the efficiency of transmission can be predicted in terms of the 
phase, amplitude and spatial distribution of the MOs on the atomic sites directly connected to the electrodes. ${ }^{55,56}$ According to the orbital symmetry rules derived by Yoshizawa and co-workers, ${ }^{57,58}$ at an energy $E$ around the Fermi level the contribution from the frontier HOMO and LUMO orbitals is mainly responsible for Green's function, which is written as

$$
G_{l r}^{m o l}(E)=\frac{C_{l, H O M O} C_{r, H O M O}^{\star}}{E-\varepsilon_{H O M O}}+\frac{C_{l, L U M O} C_{r, L U M O}^{\star}}{E-\varepsilon_{L U M O}}
$$

where $C_{l / r, \text { HOMO }}$ and $C_{l / r, L U M O}$ are the MO coefficients of the HOMO and LUMO at the connecting sites $l$ and $r$ and $\varepsilon_{H O M O / L U M O}$ are the HOMO/LUMO orbital energies. Since the signs of denominators are opposite in Eq. 6 for the energy $E$ located between the HOMO and LUMO. Hence, QI is determined by the signs of the numerators of the two terms. When the numerators have same sign, the contribution from HOMO and LUMO will cancel each other and results in DQI. On contrary, if numerators have opposite signs, CQI is predicted. However, if all four involved amplitudes $C_{l, H O M O}, C_{r, \text { HOMO }}^{\star}, C_{l, L U M O}, C_{r, L U M O}^{\star}$ do not have significant magnitude, then it results in an inefficient transmission, but not necessarily the typical DQI.
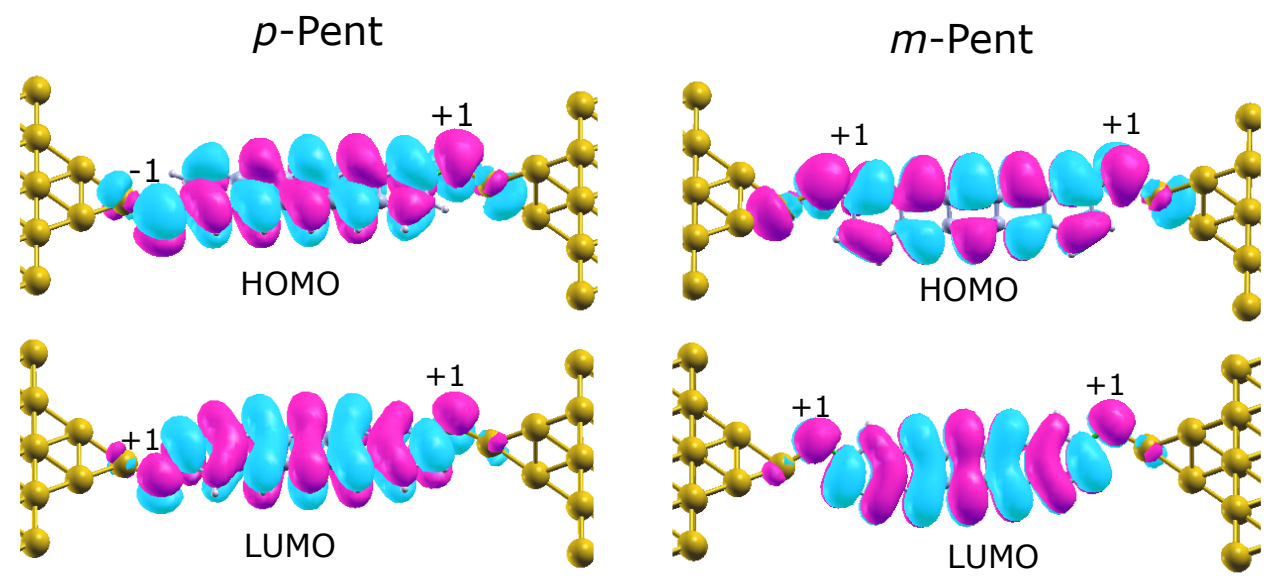

Figure 5: Spatial distribution of MPSH frontier orbitals for $p$-Pent and $m$-Pent in molecular junctions. Pink and cyan colors denote the positive and negative parity of the molecular orbitals with isovalue 0.02 a.u. and is represented by +1 and -1 marked over the anchoring $\mathrm{S}$ atoms.

The spatial distribution of the frontier MPSH orbitals for $p$-Pent and $m$-Pent are shown in 
Fig. 5. The pink and cyan colors represent the positive and negative parity of the MOs and is denoted by +1 and -1 on sulphur atoms respectively. For $p$-Pent, the signs of the coefficients at two sulphur atoms are opposite for HOMO but are same for LUMO. Thus, according to Eq. 6, the HOMO coefficients product is negative and LUMO coefficients product is positive, this results in opposite signs of two numerators and hence CQI for $p$-Pent. However, for $m$-Pent, the signs of the coefficients at two sulphur atoms are same for both HOMO and LUMO, thus the contributions from HOMO and LUMO will cancel each other and result in DQI for $m$-Pent.

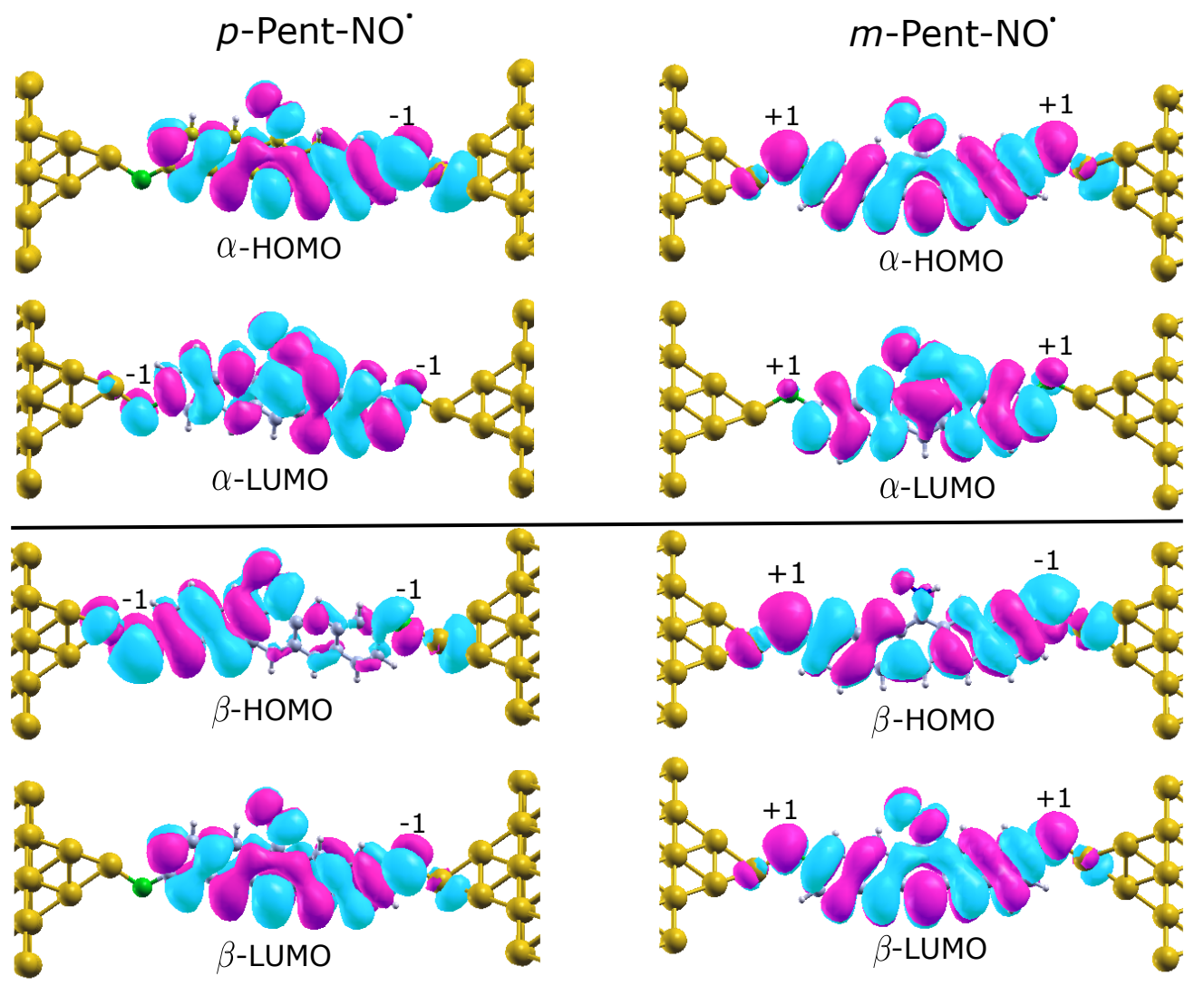

Figure 6: Spatial distribution of spin-up (top panel) and spin-down (bottom panel) MPSH frontier orbitals for $p$-Pent-NO and $m$-Pent-NO in molecular junctions. Pink and cyan colors denote the positive and negative parity of the molecular orbitals with isovalue 0.02 a.u. and is represented by +1 and -1 marked over the anchoring $\mathrm{S}$ atoms. Low orbital density on terminal S-atoms of $p$-Pent-NO reveals inefficient transmission. For $m$-Pent-NO;, DQI and CQI is predicted for spin-up and spin-down electrons respectively.

For radical molecular junctions, due to the presence of non-bonding MO near Fermi 
energy, the orbitals of one of the spin configuration do not approximate the exact orbital symmetry. As a result, the orbital symmetry rules might not be fully applicable for both the spin configurations. Here, this also calls for the rules that can reliably capture CQI and DQI in spin polarized molecular junctions with localized radical centers. Nevertheless, when the orbital coefficients of HOMO and LUMO have same sign at the contact positions, a cancellation will always occur somewhere in the window of interest, despite of the asymmetrical orbital distribution. Fig. 6 illustrates the spin-up and spin-down frontier MPSH MOs of $p$-Pent-NO* and $m$-Pent-NO in junction. For $p$-Pent-NO; both $\alpha$-HOMO and $\beta$-LUMO exhibits negligible orbital density at the left terminal S-atom indicating weak coupling to left electrode and hence inefficient transmission. The significance of orbital density on terminal atoms in determining the charge transport through a device was also proposed by Ernzerhof and Rocheleau. ${ }^{59}$ While for $m$-Pent-NO; although the orbital density on left S-atom of $\alpha$-LUMO is small, however, the signs of the coefficients at two sulphur atoms are positive for both $\alpha$-HOMO and $\alpha$-LUMO. This clearly indicates the partial cancellation of the two terms in Eq. 6 and hence signatures of DQI in spin-up configuration of $m$-Pent-NO: For spin-down electrons, all the terminal S-atoms bear significant amplitude with the product of the coefficients being negative for $\mathrm{HOMO}$ and positive for LUMO giving clear prediction of CQI in spin-down configuration of $m$-Pent-NO:

\subsubsection{Transmission Eigenchannels}

Transmission Eigenchannels (EC) provide an intuitive picture of the electron transport by breaking the transmission into non-mixing channels where each EC has a well defined transmission probability. Fig. 7 illustrates the ECs for $p$-Pent and $m$-Pent. For $p$-Pent, both the pathways connecting left and right electrode are equally conductive with strong terminal coupling at both the ends for both Left-EC and Right-EC. However, for $m$-Pent, only shorter pathway connecting left and right electrode is conductive with relatively weaker coupling at one of the terminating end. Thus, both the Eigenchannels of $m$-Pent are only partially open 
for conduction, in contrast to fully conductive channels of $p$-Pent, which strongly supports the lower conductance of $m$-Pent as compared to $p$-Pent attributed to QI effects.
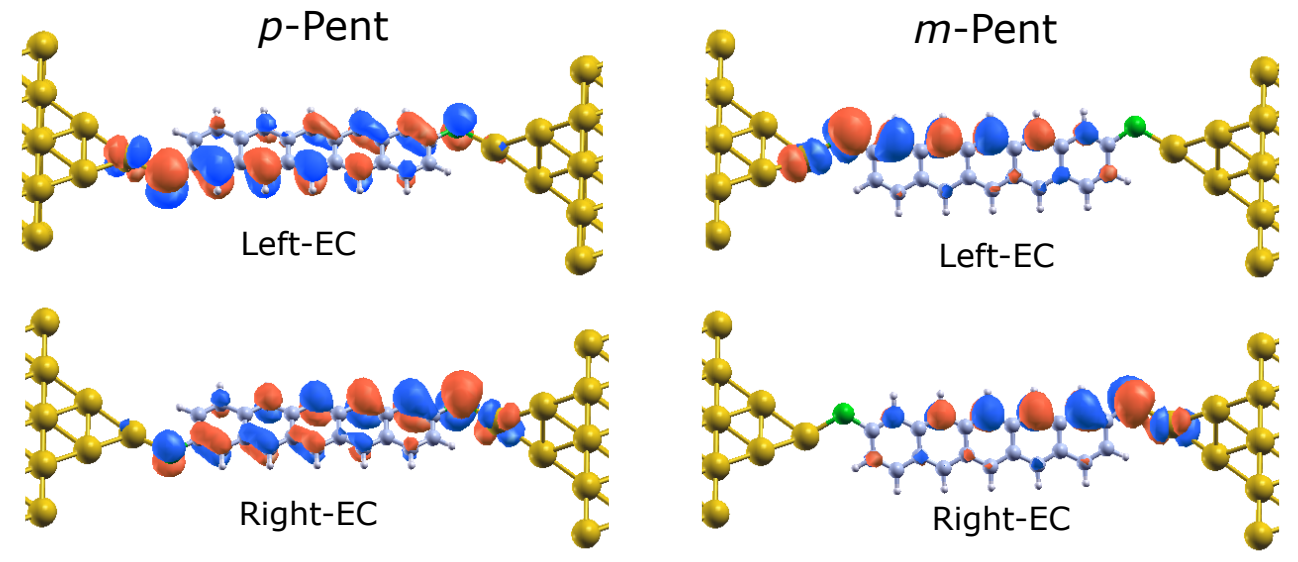

Figure 7: Transmission Eigenchannels (EC) of $p$-Pent and $m$-Pent. Terminal coupling is weak for $m$-Pent than $p$-Pent.

For $p$-Pent-NO and $m$-Pent-NO; both the spin ECs are illustrated in Fig. 8. In case of $p$-Pent-NO the EC is either blocked by radical center or is partially conductive due to weak terminal coupling. In contrast, $m$-Pent-NO' exhibits fully conductive channels with strong terminal coupling for both the spin channels. Thus clearly indicating higher conductance of $m$-Pent-NO than $p$-Pent-NO*. Apart from this, spin-up ECs are more conductive than spin-down channels for both $p$-Pent-NO* and $m$-Pent-NO* except $\alpha$-Left-EC of $p$-Pent-NO*. The higher conductance of spin-up electrons as compared to the spin-down electrons is also revealed from transmission spectra (in Fig. 4) with $\alpha$-HOMO lying near Fermi energy than $\beta$-HOMO, thus, indicating perfect spin filtering in radical molecular junctions. 


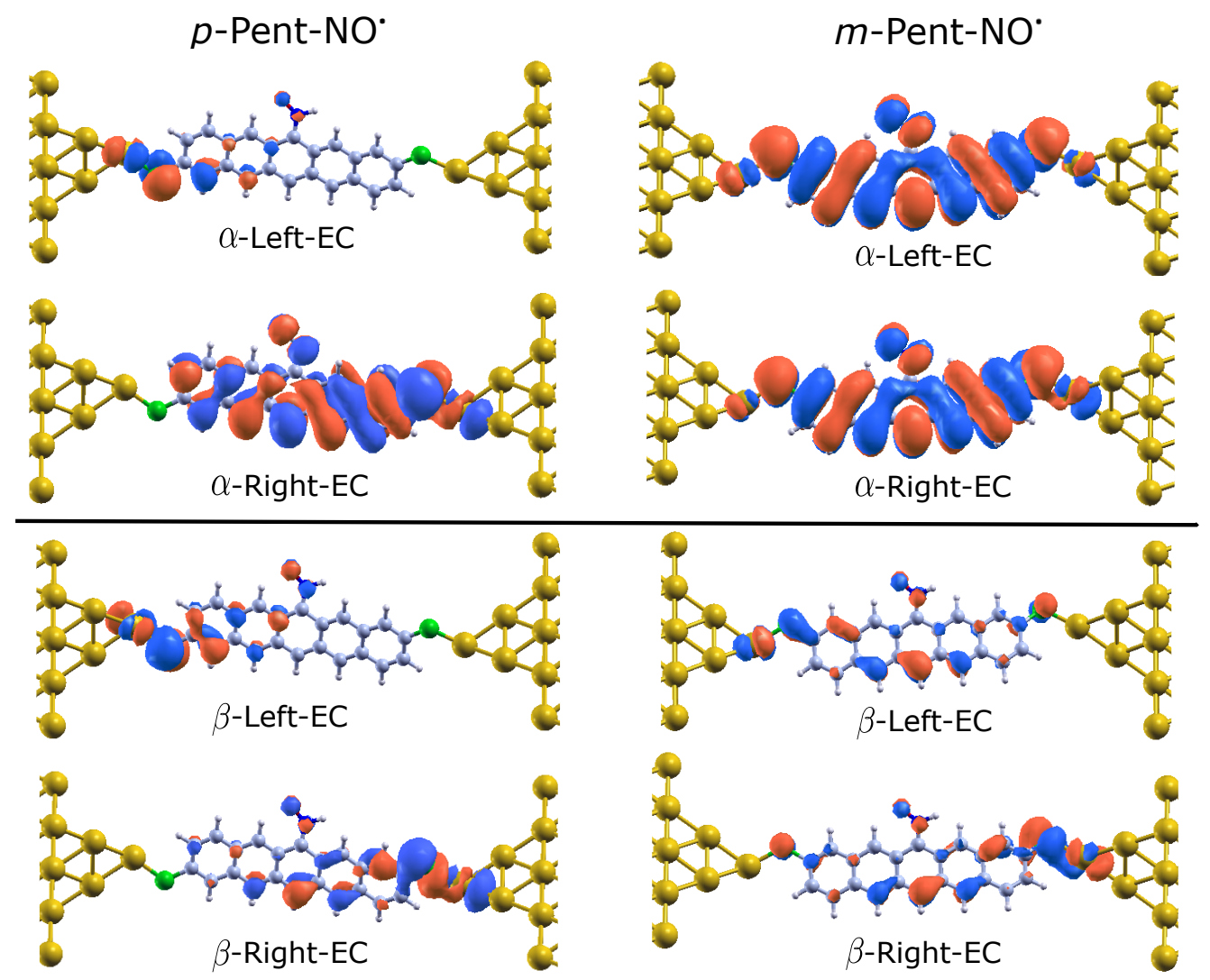

Figure 8: Transmission Eigenchannels (EC) for spin-up (top panel) and spin-down (bottom panel) channels of $p$-Pent-NO and $m$-Pent-NO. ECs of $p$-Pent-NO are either blocked by radical center or by terminal thiol group.

\subsubsection{Local Transmission Analysis}

In order to get further insight into the spin-resolved transport properties of radical molecular junctions, we evaluated the local transmission maps for the transmission spectra associated with cluster approach (shown in SI) using ARTAIOS code. From local transmission plots one can obtain a qualitative understanding of how the electron flows through the molecule from contact to contact. In these plots, the local transmission components are represented by arrows along molecular geometry in such a way that diameter of arrow is proportional to the value of local transmission between pair of atoms. Blue arrows indicate local transport contributions in the same direction as of overall current, i.e., when the transmission from the first atom to second atom is positive and the second atom lies further along the transport 
direction (left to right) thereby contributing to the current, whereas the red arrows gives the components corresponding to the negative transmission, thereby reducing the net current. An arrow is only drawn when the magnitude of local transmission is at least $10 \%$ of the maximum local transmission at that energy. Previously, Solomon et al. demonstrated how QI effects can be characterized from the description of local transmissions. The dips in transmission spectra of m-Benzene associated with QI were characterized by ring current reversal, while none of these features were observed for p-Benzene. ${ }^{43}$ These striking features of cyclic currents are also connected to signatures of QI in a recent report by Stuyver et al. ${ }^{60}$
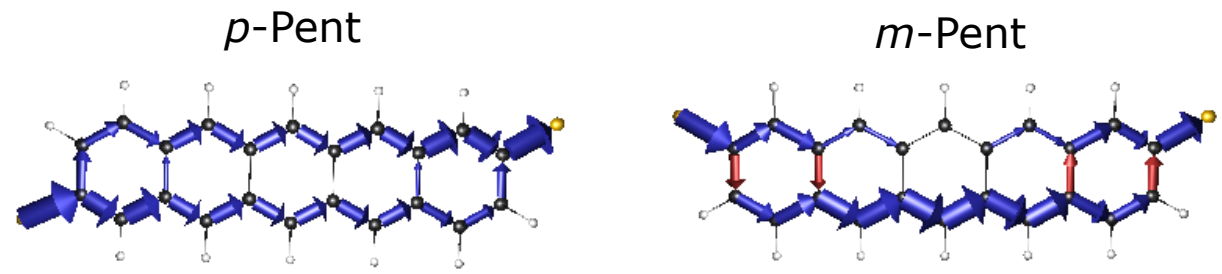

Figure 9: Local transport contributions in $p$-Pent and $m$-Pent associated with QI dips at Fermi level. The diameter of the arrow is proportional to the magnitude of local transmission contribution, which are depicted only if they are $10 \%$ of the maximum local transmission between two atoms. Arrows in the transport direction are colored blue and the ones opposite to it are red.

Fig. 9 shows local transport contributions for $p$-Pent and $m$-Pent at Fermi energy where QI features are prominent. Both $p$-Pent and $m$-Pent exhibits currents in the terminal benzene rings wherein three consecutive arrows are circulating clockwise $(\mathrm{CW})$ and the remaining three are circulating in anti-clockwise (ACW) direction. For $p$-Pent, CW and ACW pathways are interfering constructively (with both blue colored arrows meeting each other). While for $m$-Pent, the conjoint of blue and red colored arrows reveals destructive interference between CW and ACW pathways, thus reducing the net transmission.

For $p$-Pent-NO' and $m$-Pent-NO; the local transmissions are plotted at the energies close to QI dips around Fermi level and are illustrated in Fig. 10. For p-Pent-NO; the conjoint of red and blue arrows in the terminal and central benzene rings of spin-up and spin-down electrons respectively reveals the signatures of DQI. On the other hand, $m$-Pent-NO' yields constructively interfering pathways. This scenario is in complete contrast to the one observed 


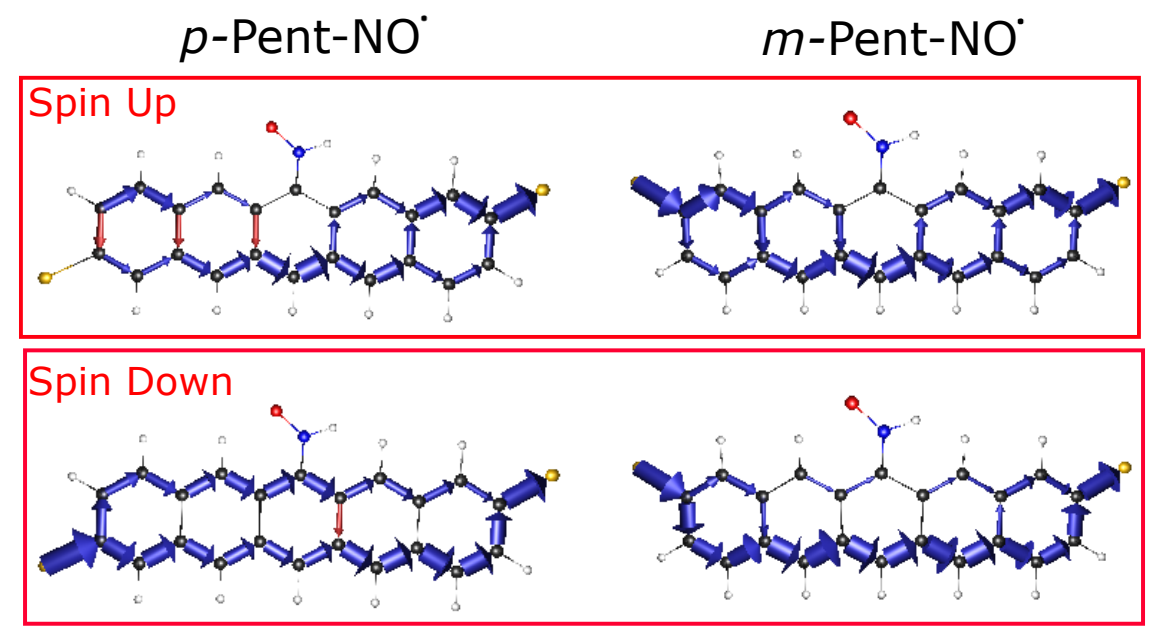

Figure 10: Local transport contributions in $p$-Pent-NO and $m$-Pent-NO at energies close to QI dips near Fermi level.

for parent pentacene and vividly supports the anomalous behavior of QI effects by attachment of radical. Besides the QI features, the local transport plots also suggest that conducting electrons go through the pentacene backbone while not directly interacting with radical. Thus, although there is no direct participation of radical in electron transport, however, significant spin polarization is induced by radical owing to the complete delocalization of spin density on the pentacene backbone. The local transport plots for other radicals at different energies are presented in SI.

Such breakdown of interference rules where the conductance of meta coupled molecules can be high is also witnessed in few other reports also. ${ }^{61,62}$ A recent report by Garner et al. hypothesized that if the $\sigma$ system of the molecule is more strongly coupled to the electrodes than the $\pi$ system, DQI effects can be masked by $\sigma$-conductance. ${ }^{63,64}$ But here for the radical system, due to the presence of unpaired electron residing in $\pi$-orbital, $\sigma$-systems cannot be the dominant contributor to the transport. In addition the quenching of QI effects have also been extensively discussed in terms of the dephasing introduced by electron-phonon interactions. ${ }^{65}$ Apart from the inelastic scattering arising from interaction between conducting electrons and molecular vibrations, here, for the junctions exhibiting localized spin centres, the spin-spin interaction between the conducting electron and localized electron could be the 
other dominating dephasing factor. Since, the molecular junctions are subjected to implicit dephasing environmental conditions during experiments, the intrinsic mechanism of such dephasing effects needs to be investigated in details.

\section{Conclusion}

We theoretically investigated the QI effects in spin polarized junctions and realized the anomalous behavior of QI stemming from localized radical spins in molecular junctions. The exchange splittings split the energy levels in the radical junctions and invoke the anomaly in QI patterns in such a way that for meta connected radicals, DQI dip observed in parent $m$-Pent is shifted to higher energy for one of the spin orientation, thereby increasing the conductance at Fermi level, while it completely transforms to a CQI feature for other spin orientation. For para connected radicals, one of the spin orientation yield usual CQI feature as of parent $p$-Pent but accompanied with decreased conductance at Fermi level, however, other spin orientation results in complete crossover to DQI feature. In conclusion, meta connected radicals yields higher conductance than para ones for both spin orientations, which is in complete contrast to non-magnetic pentacene as well as non-radical analogues of all the radicals under study.

We further demonstrate that for the non-magnetic acenes, QI effects intensify with increasing molecular length in such a way that most evident signatures of QI are observed in pentacene. Apart from this, the absence of magnetic centers results in spin-independent transport in acene based junctions. However, high spin filtering efficiency is observed for all the para and meta connected radical systems owing to the substantial delocalization of spin density on the entire pentacene backbone. Thus, we believe that our findings provide a potential approach to facilitate future design attempts of single localized spin molecular junctions. 


\section{Acknowledgement}

We are thankful to Prof. Dr Carmen Herrmann for providing the transport code ARTAIOS and Rameshwar L. Kumawat for various helpful discussions. Financial support from the Department of Science and Technology through SERB-ECR project no. ECR/2016/000362 and Indo-Sweden joint project no. DST/INT/SWD/VR/P-01/2016 and computational resources obtained from CDAC-Pune are gratefully acknowledged.

Supporting Information Available: Quantum interference in parent acenes, Molecular PDOS analysis, Energy level diagram and HOMO-LUMO gap for molecules in gas phase and in junction, MPSH eigenstates and transmission eigenchannels.

This material is available free of charge via the Internet at http:// pubs.acs.org.

\section{References}

(1) Hirose, K.; Kobayashi, N. Quantum transport calculations for nanosystems; Jenny Stanford Publishing, 2014.

(2) Tao, N. J. Nanoscience And Technology: A Collection of Reviews from Nature Journals; World Scientific, 2010.

(3) Rocha, A. R.; Garcia-Suarez, V. M.; Bailey, S. W.; Lambert, C. J.; Ferrer, J.; Sanvito, S. Towards molecular spintronics. Nat. Mater. 2005, 4, 335-339.

(4) Mannini, M.; Pineider, F.; Sainctavit, P.; Danieli, C.; Otero, E.; Sciancalepore, C.; Talarico, A. M.; Arrio, M.-A.; Cornia, A.; Gatteschi, D. et al. Magnetic memory of a single-molecule quantum magnet wired to a gold surface. Nat. Mater. 2009, 8, 194-197.

(5) Dediu, V. A.; Hueso, L. E.; Bergenti, I.; Taliani, C. Spin routes in organic semiconductors. Nat. Mater. 2009, 8, 707-716.

(6) Barraud, C.; Seneor, P.; Mattana, R.; Fusil, S.; Bouzehouane, K.; Deranlot, C.; 
Graziosi, P.; Hueso, L.; Bergenti, I.; Dediu, V. et al. Unravelling the role of the interface for spin injection into organic semiconductors. Nat. Phys 2010, 6, 615-620.

(7) Herrmann, C.; Solomon, G. C.; Ratner, M. A. Organic radicals as spin filters. J. Am. Chem. Soc. 2010, 132, 3682-3684.

(8) Herrmann, C.; Solomon, G. C.; Ratner, M. A. Designing organic spin filters in the coherent tunneling regime. J. Chem. Phys. 2011, 134, 224306.

(9) Lach, S.; Altenhof, A.; Tarafder, K.; Schmitt, F.; Ali, M. E.; Vogel, M.; Sauther, J.; Oppeneer, P. M.; Ziegler, C. Metal-organic hybrid interface states of a ferromagnet/organic semiconductor hybrid junction as basis for engineering spin injection in organic spintronics. Adv. Funct. Mater 2012, 22, 989-997.

(10) Smeu, M.; DiLabio, G. A. Calculations of electron transport through simple $\pi$-and $\sigma$-type radicals. J. Phys. Chem. C 2010, 114, 17874-17879.

(11) Shil, S.; Bhattacharya, D.; Misra, A.; Klein, D. J. A high-spin organic diradical as a spin filter. Phys. Chem. Chem. Phys. 2015, 17, 23378-23383.

(12) Su, T. A.; Neupane, M.; Steigerwald, M. L.; Venkataraman, L.; Nuckolls, C. Chemical principles of single-molecule electronics. Nat. Rev. Mater. 2016, 1, 16002.

(13) Li, X.; Tan, Z.; Huang, X.; Bai, J.; Liu, J.; Hong, W. Experimental investigation of quantum interference in the charge transport through molecular architectures. J. Mater. Chem. C 2019, 7, 12790-12808.

(14) Hansen, T.; Solomon, G. C.; Andrews, D. Q.; Ratner, M. A. Interfering pathways in benzene: An analytical treatment. J. Chem. Phys 2009, 131, 194704.

(15) Taniguchi, M.; Tsutsui, M.; Mogi, R.; Sugawara, T.; Tsuji, Y.; Yoshizawa, K.; Kawai, T. Dependence of single-molecule conductance on molecule junction symmetry. J. Am. Chem. Soc. 2011, 133, 11426-11429. 
(16) Lambert, C. Basic concepts of quantum interference and electron transport in singlemolecule electronics. Chem. Soc. Rev. 2015, 44, 875-888.

(17) Alanazy, A. e. a. Cross-conjugation increases the conductance of meta-connected fluorenones. Nanoscale 2019, 11, 13720-13724.

(18) Guédon, C. M.; Valkenier, H.; Markussen, T.; Thygesen, K. S.; Hummelen, J. C.; Van Der Molen, S. J. Observation of quantum interference in molecular charge transport. Nat. Nanotechnol. 2012, 7, 305-309.

(19) Manrique, D. Z.; Huang, C.; Baghernejad, M.; Zhao, X.; Al-Owaedi, O. A.; Sadeghi, H.; Kaliginedi, V.; Hong, W.; Gulcur, M.; Wandlowski, T. et al. A quantum circuit rule for interference effects in single-molecule electrical junctions. Nat. Commun 2015, 6, 1-8.

(20) Sangtarash, S.; Sadeghi, H.; Lambert, C. J. Exploring quantum interference in heteroatom-substituted graphene-like molecules. Nanoscale 2016, 8, 13199-13205.

(21) Markussen, T.; Stadler, R.; Thygesen, K. S. Graphical prediction of quantum interferenceinduced transmission nodes in functionalized organic molecules. Phys. Chem. Chem. Phys 2011, 13, 14311-14317.

(22) Li, Y.; Buerkle, M.; Li, G.; Rostamian, A.; Wang, H.; Wang, Z.; Bowler, D. R.; Miyazaki, T.; Xiang, L.; Asai, Y. et al. Gate controlling of quantum interference and direct observation of anti-resonances in single molecule charge transport. Nat. Mater. 2019, 18, 357-363.

(23) Arroyo, C. R.; Tarkuc, S.; Frisenda, R.; Seldenthuis, J. S.; Woerde, C. H.; Eelkema, R.; Grozema, F. C.; Van Der Zant, H. S. Signatures of quantum interference effects on charge transport through a single benzene ring. Angew. Chem 2013, 52, 3152-3155.

(24) Markussen, T.; Stadler, R.; Thygesen, K. S. The relation between structure and quantum interference in single molecule junctions. Nano Lett. 2010, 10, 4260-4265. 
(25) Berritta, M.; Manrique, D. Z.; Lambert, C. J. Interplay between quantum interference and conformational fluctuations in single-molecule break junctions. Nanoscale 2015, 7, 1096-1101.

(26) Valli, A.; Amaricci, A.; Brosco, V.; Capone, M. Quantum interference assisted spin filtering in graphene nanoflakes. Nano Lett. 2018, 18, 2158-2164.

(27) Valli, A.; Amaricci, A.; Brosco, V.; Capone, M. Interplay between destructive quantum interference and symmetry-breaking phenomena in graphene quantum junctions. Phys. Rev. B 2019, 100, 075118.

(28) Bergfield, J. P.; Solomon, G. C.; Stafford, C. A.; Ratner, M. A. Novel quantum interference effects in transport through molecular radicals. Nano Lett. 2011, 11, 27592764 .

(29) Pal, A. N.; Li, D.; Sarkar, S.; Chakrabarti, S.; Vilan, A.; Kronik, L.; Smogunov, A.; Tal, O. Nonmagnetic single-molecule spin-filter based on quantum interference. Nat. Commun 2019, 10, 1-8.

(30) Li, D.; Banerjee, R.; Mondal, S.; Maliyov, I.; Romanova, M.; Dappe, Y. J.; Smogunov, A. Symmetry aspects of spin filtering in molecular junctions: Hybridization and quantum interference effects. Phys. Rev. B 2019, 99, 115403.

(31) Saraiva-Souza, A.; Smeu, M.; Zhang, L.; Souza Filho, A. G.; Guo, H.; Ratner, M. A. Molecular spintronics: Destructive quantum interference controlled by a gate. J. Am. Chem. Soc. 2014, 136, 15065-15071.

(32) Taylor, J.; Guo, H.; Wang, J. Ab initio modeling of quantum transport properties of molecular electronic devices. Phys. Rev. B 2001, 63, 245407.

(33) Toher, C.; Filippetti, A.; Sanvito, S.; Burke, K. Self-interaction errors in densityfunctional calculations of electronic transport. Phys. Rev. Lett. 2005, 95, 146402. 
(34) Kim, W. Y.; Choi, Y. C.; Min, S. K.; Cho, Y.; Kim, K. S. Application of quantum chemistry to nanotechnology: electron and spin transport in molecular devices. Chem. Soc. Rev 2009, 38, 2319-2333.

(35) Soler, J. M.; Artacho, E.; Gale, J. D.; García, A.; Junquera, J.; Ordejón, P.; SánchezPortal, D. The SIESTA method for ab initio order-N materials simulation. J. Phys. Condens. Matter 2002, 14, 2745.

(36) Neese, F. The ORCA program system. Wiley Interdiscip. Rev. Comput. Mol. Sci 2012, 2, 73-78.

(37) Stokbro, K.; Taylor, J.; Brandbyge, M.; Ordejon, P. TranSIESTA: a spice for molecular electronics. Ann. N. Y. Acad. Sci 2003, 1006, 212-226.

(38) Troullier, N.; Martins, J. L. Efficient pseudopotentials for plane-wave calculations. Phys. Rev. B 1991, 43, 1993.

(39) Deffner, M.; Gross, L.; Steenbock, T.; Voigt, B.; Solomon, G.; Herrmann, C. Artaios - a code for postprocessing quantum chemical electronic structure calculations. 2010.

(40) Herrmann, C.; Solomon, G. C.; Subotnik, J. E.; Mujica, V.; Ratner, M. A. Ghost transmission: How large basis sets can make electron transport calculations worse. $J$. Chem. Phys 2010, 132, 024103.

(41) Datta, S. Nanoscale device modeling: the Green's function method. Superlattices Microstruct 2000, 28, 253-278.

(42) Paulsson, M.; Brandbyge, M. Transmission eigenchannels from nonequilibrium Green's functions. Phys. Rev. B 2007, 76, 115117.

(43) Solomon, G. C.; Herrmann, C.; Hansen, T.; Mujica, V.; Ratner, M. A. Exploring local currents in molecular junctions. Nat. Chem. 2010, 2, 223. 
(44) Kamiński, W.; Topolnicki, R.; Hapala, P.; Jelínek, P.; Kucharczyk, R. Tuning the conductance of benzene-based single-molecule junctions. Org. Electron 2016, 34, 254261.

(45) Xiao, X.; Xu, B.; Tao, N. J. Measurement of single molecule conductance: Benzenedithiol and benzenedimethanethiol. Nano Lett. 2004, 4, 267-271.

(46) Kiguchi, M.; Nakamura, H.; Takahashi, Y.; Takahashi, T.; Ohto, T. Effect of anchoring group position on formation and conductance of a single disubstituted benzene molecule bridging Au electrodes: change of conductive molecular orbital and electron pathway. $J$. Phys. Chem. C 2010, 114, 22254-22261.

(47) Solomon, G. C.; Andrews, D. Q.; Hansen, T.; Goldsmith, R. H.; Wasielewski, M. R.; Van Duyne, R. P.; Ratner, M. A. Understanding quantum interference in coherent molecular conduction. J. Chem. Phys 2008, 129, 054701.

(48) Rodriguez-Gonzalez, S.; Xie, Z.; Galangau, O.; Selvanathan, P.; Norel, L.; Van Dyck, C.; Costuas, K.; Frisbie, C.; Rigaut, S.; Cornil, J. Homo level pinning in molecular junctions: Joint theoretical and experimental evidence. J. Phys. Chem. Lett. 2018, 9, 2394-2403.

(49) Kaur, P.; Ali, M., et al. First Principle Investigations of Long-range Magnetic Exchange Interactions via Polyacene Coupler. 2020, doi: https://doi.org/10.26434/chemrxiv . 12776222.v1.

(50) Lambert, C. J.; Baghernejad, M.; Yang, Y.; Al Owaedi, O.; Aeshchi, Y.; Zeng, B.F.; Dawood, Z.; Li, X.; Liu, J.; Shi, J. et al. Constructive Quantum Interference in Single-Molecule Benzodichalcogenophene Junctions. Chem. Eur. J. 2020, 10.1002/chem.201905878.

(51) Bajaj, A.; Ali, M. E. Tuning of spin-polarized current in high spin organic molecules. Physica B: Condense Matter 2020, 412396. 
(52) Larade, B.; Taylor, J.; Zheng, Q.; Mehrez, H.; Pomorski, P.; Guo, H. Renormalized molecular levels in a Sc 3 N@ C 80 molecular electronic device. Phys. Rev. B 2001, 64, 195402.

(53) Stokbro, K.; Taylor, J.; Brandbyge, M.; Mozos, J.-L.; Ordejon, P. Theoretical study of the nonlinear conductance of Di-thiol benzene coupled to Au ( $\left.\begin{array}{lll}1 & 1 & 1\end{array}\right)$ surfaces via thiol and thiolate bonds. Comput. Mater. Sci 2003, 27, 151-160.

(54) Lambert, C. J.; Liu, S.-X. A magic ratio rule for beginners: a chemist's guide to quantum interference in molecules. Chem. Eur. J. 2018, 24, 4193-4201.

(55) Zhao, X.; Geskin, V.; Stadler, R. Destructive quantum interference in electron transport: A reconciliation of the molecular orbital and the atomic orbital perspective. J. Chem. Phys 2017, 146, 092308.

(56) Tsuji, Y.; Hoffmann, R. Frontier orbital control of molecular conductance and its switching. Angew. Chem. 2014, 53, 4093-4097.

(57) Yoshizawa, K. An orbital rule for electron transport in molecules. Acc. Chem. Res 2012, 45, 1612-1621.

(58) Yoshizawa, K.; Tada, T.; Staykov, A. Orbital views of the electron transport in molecular devices. J. Am. Chem. Soc 2008, 130, 9406-9413.

(59) Rocheleau, P.; Ernzerhof, M. Molecular conductance obtained in terms of orbital densities and response functions. J. Chem. Phys 2009, 130, 184704.

(60) Stuyver, T.; Blotwijk, N.; Fias, S.; Geerlings, P.; De Proft, F. Exploring Electrical Currents through Nanographenes: Visualization and Tuning of the through-Bond Transmission Paths. ChemPhysChem 2017, 18, 3012-3022.

(61) Xia, J.; Capozzi, B.; Wei, S.; Strange, M.; Batra, A.; Moreno, J. R.; Amir, R. J.; 
Amir, E.; Solomon, G. C.; Venkataraman, L. et al. Breakdown of interference rules in azulene, a nonalternant hydrocarbon. Nano Lett. 2014, 14, 2941-2945.

(62) Li, S.; Yu, H.; Schwieter, K.; Chen, K.; Li, B.; Liu, Y.; Moore, J. S.; Schroeder, C. M. Charge transport and quantum interference effects in oxazole-terminated conjugated oligomers. J. Am. Chem. Soc. 2019, 141, 16079-16084.

(63) Garner, M. H.; Li, H.; Chen, Y.; Su, T. A.; Shangguan, Z.; Paley, D. W.; Liu, T.; Ng, F.; Li, H.; Xiao, S. et al. Comprehensive suppression of single-molecule conductance using destructive $\sigma$-interference. Nature 2018, 558, 415-419.

(64) Borges, A.; Xia, J.; Liu, S. H.; Venkataraman, L.; Solomon, G. C. The role of throughspace interactions in modulating constructive and destructive interference effects in benzene. Nano Lett. 2017, 17, 4436-4442.

(65) Maassen, J.; Zahid, F.; Guo, H. Effects of dephasing in molecular transport junctions using atomistic first principles. Phys. Rev. B 2009, 80, 125423. 
Graphical TOC Entry

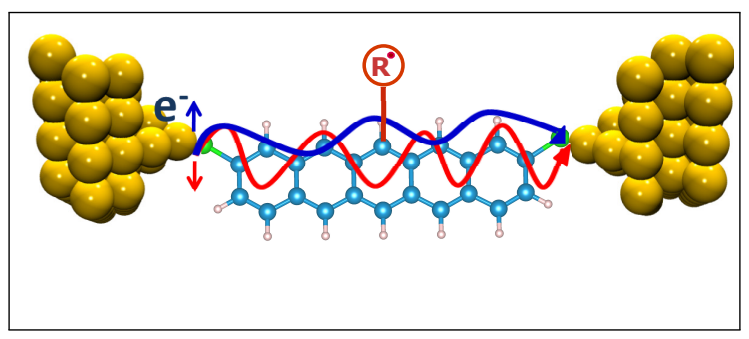

